\title{
Appraisal of caesarean section incidence and indications at manipal teaching hospital, Pokhara, Nepal
}

\author{
Aashika Shrestha', Junu Shrestha' ${ }^{2}$, Sangeeta Devi Gurung ${ }^{3}$ \\ ${ }^{1}$ Lecturer, ${ }^{2}$ Associate Professor, ${ }^{3}$ Assistant Professor, Department of Obstetrics and Gynecology, Manipal College of \\ Medical Sciences, Pokhara, Nepal
}

Background: Worldwide lower segment caesarean section (LSCS) is the most commonly performed obstetric operation. Though it's a lifesaving procedure it is sometime associated with both short and long term risk which can extend many years beyond current delivery and affect health of mother, fetus and future pregnancy. Aims and Objective: The study aims to examine the incidence and indication of caesarean section at Manipal teaching hospital. Materials and Methods: A retrospective descriptive study of 2140 cases was carried out at a teaching hospital in Pokhara, Nepal. Data regarding total number of vaginal delivery and caesarean section performed in 1 year duration from August 2019 to July 2020 was obtained from the operation record book and medical record section of hospital. Other information regarding age, parity, gestational period and indication of cesarean section were obtained and depicted in tabulated form. The collected secondary data has been analyzed employing frequencies and percentage. Results: During the one year study period, 2140 deliveries were made out of which 1170 (54.7\%) cases underwent caesarean section. Out of those cases $78.8 \%$ of women underwent emergency LSCS. Most of the women were in the age group of $21-30$ years $(69.2 \%)$. In $81.2 \%$ cases caesarean was done between $38-42$ weeks gestation. $52.3 \%$ of cases were multigravida, $43.8 \%$ were primigravida and only $3.1 \%$ were grand multigravida. Most common indication of caesarean were fetal distress (23.7\%), previous LSCS (23\%), cephalopelvic disproportion (15.6\%), oligohydramnios (11\%) followed by breech, preeclampsia, eclampsia and non-progress of labour.Conclusion: This study revealed that the incidence of caesarean section was high in the hospital and majority of the patients who underwent caesarean section had an emergency caesarean section. The commonest indications were fetal distress and repeat cesarean section. The issue requires further investigation to identify reasons for high CS rate and raises necessity of policy guidelines and intervention in order to prevent possible unjustified use of surgical delivery.
Access this article online

Website:

http://nepjol.info/index.php/AJMS

DOI: 10.3126/ajms.v12i1.30846

E-ISSN: 2091-0576

P-ISSN: $2467-9100$

Copyright (c) 2021 Asian Journal of Medical Sciences

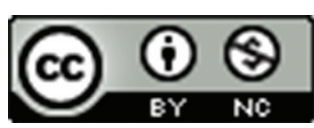

This work is licensed under a Creative Commons Attribution-NonCommercial 4.0 International License.

Key words: Cesarean section; Incidence; Indications; Nepal

\section{INTRODUCTION}

Worldwide lower segment caesarean section is the most commonly performed obstetric operation. It is a lifesaving procedure when certain complication arises which has a detrimental health effect either to the mother or fetus both during pregnancy and at the time of birth. However, it is also associated with both short and long term risk which has an impact not only in current delivery but also extends in future and affects the health of the woman, her child, and future pregnancies. These risks are higher in women with limited access to comprehensive obstetric care. ${ }^{1}$

Worldwide the rate of caesarean section is increasing at an alarming rate, despite the lack of evidence which supports substantial maternal and perinatal benefits with CS rates higher than a certain threshold, and some of the studies even showing a link between increasing CS rates and poorer outcomes. ${ }^{2-4}$ 
The World Health Organization (WHO) has suggested a caesarean rate between $10 \%$ and $15 \%$ and if the rate goes above $15 \%$ it implies an unnecessary and unjustified use of surgical delivery, whereas rate below $5 \%$ was associated with population's lack of access to medical technology. ${ }^{5}$

Contrary to WHO statement and lots of recommendations and warnings about risk of caesarean, its rate has been constantly rising across the globe reaching to a epidemic proportion in some countries and showing no sign of slowing down. ${ }^{6-7}$

The reasons for this increase can be multifactorial like Changes in maternal characteristics and professional practice styles, increasing malpractice pressure, social and cultural factors, improvement of surgical and anesthetic techniques, reduction of the postoperative complications and the perception of greater safety during the procedure.

Ensuring safe pregnancy and motherhood occupies a pivotal role and is considered as one of the key issues in the reproductive health policies [Table 1]. Caesarean section is associated with maternal and perinatal risk. As identified by various prior studies, its rate has increased dramatically over the past three decades both in the developed and in the developing world. In this context, it is important to empirically investigate the prevalence and examine its indications in Nepal. As few studies have been conducted on the topic in Nepal, the study aimed to empirically examine the incidence and indications of cesarean section at Manipal Teaching Hospital in Pokhara, Nepal. The study results will be useful for informing health authorities and provide a basis for formulation of health policies and strategies to control the cesarean section rate.

\section{MATERIALS AND METHODS}

This descriptive cross-sectional study is a hospital based retrospective study conducted at the Department of Obstetrics and Gynecology, Manipal Teaching Hospital, Pokhara. It is a tertiary care hospital of province number 4 where all kind of obstetrics related conditions are dealt with multidisciplinary approach. Service is provided for both booked as well as referral cases. Referrals are from local as well as peripheral hospitals. Study duration was of 1 year from August 2019 to July 2020.

Data was collected from operation record book as well as medical record department of hospital. Data was analyzed using SPSS version 25 and frequencies as well as percentages were calculated. Total number of deliveries both vaginal and caesarean section obtained and incidence of caesarean calculated. Other variables like type of caesarean, age, parity, gestational age and indication of LSCS was analyzed. Ethical approval was taken from respective authority.

\section{RESULTS}

The profile of caesarean section exhibits that out of the total 2140 cases, $1170(54.7 \%)$ of the patients underwent caesarean section. Most of the patients are young, multigravida and have gestation period of 38-42 weeks. The results depict that most of the women i.e. $69.2 \%$ belonged to age group 21-30 years followed by $20 \%$ in between $31-40$ years and $9.6 \%$ were below 20 years. Only $1.2 \%$ of women were above 40 years.

Gestational period wise $(81.2 \%)$ cases were more than 37 weeks, $14.2 \%$ were between 34-37 weeks and only $4.2 \%$ were less than 34 weeks. Similarly, Gravidity wise $43.8 \%$ women were primigravida whereas $52.3 \%$ were multigravida while grand multiparty comprise only $3.9 \%$ of cases. Hence, the patients were commonly young multigravida with gestational period of 38 to 42 weeks.

The incidence of caesarean section reveals that out of 2140 deliveries during the study period 1170 (54.7\%) patient underwent caesarean section. Majority of patient $922(78.8 \%)$ underwent emergency caesarean section and $(21.2 \%)$ had elective surgery [Table 2].

The indication of cesarean section depicts that the most common indication for performing LSCS was fetal distress $(23.7 \%)$ which included cases with meconium stained liquor, non-reassuring fetal heart rate pattern like fetal bradycardia, tachycardia, and deceleration in Cardiotocography. Next frequent indication was Previous LSCS (23\%) per se or with other added indications like CPD, breech, oligohydramnios etc. Cephalopelvic disproportion (15.6\%) included cases who had unengaged head or narrow pelvis diagnosed at the time of clinical pelvimetry before the onset of labour and cases who are diagnosed to have malposition of head or narrow pelvis during the time of labour. Oligohydramnios due to premature rupture of membrane, IUGR or isolated comprises $11 \%$ of cases.

Among the malpresentation Breech was the most common comprising of $7 \%$ cases and $1 \%$ was done for transverse lie. Hypertensive disorder of pregnancy like severe pre Eclampsia with impending features, Eclampsia remote from term or with other obstetric indications comprises $5.5 \%$ of cases. Similarly $5.5 \%$ of cases were done for Non progress of labour either due to arrest of dilatation or arrest in descent of head seen in partograph. Antepartum hemorrhage (2.8\%) included 
cases with placenta previa and abruptio placenta. Patient either underwent elective LSCS before they bleed or emergency following bleeding in case of placenta previa. Multiple pregnancy comprises $2 \%$ which included set of twins, one triplet and one quadruplet. Other $(1.2 \%)$ indications were failed induction, bad obstetric history, heart disease, pregnancy following In Vitro Fertilization, and Polyhydramnios [Table 3].

\section{DISCUSSION}

Caesarean section is in rising trend in both developed and developing countries. From obstetric point of view it is a procedure which helps to improve maternal and perinatal outcome if judiciously practiced but at the same time it has got detrimental effect both short and long term which can extend many years beyond the current delivery.

\begin{tabular}{lc}
\multicolumn{2}{l}{ Table 1: Profile of caesarean section patients } \\
\hline Socio-Demographic distribution & Number of patients \\
\hline Age (years) & \\
$\leq 20$ & $113(9.6 \%)$ \\
$21-30$ & $810(69.2 \%)$ \\
$31-40$ & $234(20 \%)$ \\
$>40$ & $13(1.2 \%)$ \\
Gestational age (weeks) & \\
$\leq 34$ & $49(4.2 \%)$ \\
$34-37$ & $171(14.6 \%)$ \\
$38-42$ & $950(81.2 \%)$ \\
Gravida & \\
1 & $512(43.8 \%)$ \\
$2-4$ & $612(52.3 \%)$ \\
$>4$ & $46(3.9 \%)$ \\
\hline
\end{tabular}

\begin{tabular}{lc} 
Table $2:$ Incidence of caesarean section \\
\hline Incidence Type & Number of patients \\
\hline Delivery type & \\
Vaginal delivery & $970(45.3 \%)$ \\
Caesarean section & $1170(54.7 \%)$ \\
Caesarean section type & \\
Elective LSCS & $248(21.2 \%)$ \\
Emergency LSCS & $922(78.8 \%)$ \\
\hline
\end{tabular}

\begin{tabular}{lc}
\multicolumn{2}{l}{ Table $3:$ Indication of cesarean section } \\
\hline Indication of LSCS & Number of patients \\
\hline Previous LSCS & $269(23 \%)$ \\
Cephalopelvic disproportion & $183(15.6 \%)$ \\
Fetal distress & $278(23.7 \%)$ \\
Oligohydramnios & $129(11.0 \%)$ \\
Breech & $82(7.0 \%)$ \\
Multiple pregnancy & $23(2.0 \%)$ \\
Antepartum hemorrhage & $33(2.8 \%)$ \\
Preeclampsia, Eclampsia & $65(5.5 \%)$ \\
Transverse lie & $11(1.0 \%)$ \\
Non progress of labour & $65(5.5 \%)$ \\
Failed induction & $17(1.4 \%)$ \\
Others & $15(1.2 \%)$ \\
\hline
\end{tabular}

WHO has considered the ideal rate of CS to be between $10 \%$ and $15 \%{ }^{5}$ Caesarean rate calculated in our study was $54.7 \%$. Ours is an established referral Centre for all high risk cases from nearby health Centre as well as peripheral health sectors. Most of the referred cases are complicated necessitating CS for maternal or fetal indications. In a study done in Kathmandu Model hospital by Poudel et $\mathrm{al}^{8}$ the rate of caesarean was $66.1 \%$ which is higher than ours. Pradhan et $\mathrm{al}^{9}$ performed a study in Patan Hospital where the incidence of CS was $41.9 \%$. In a study done by Dhakal et $\mathrm{al}^{10}$ regarding rates of CS in urban Nepal, CS rate in BPKIH, Dharan was $28.6 \%$, TU 25.4\%, Kritipur hospital $50.9 \%$, Kathmandu medical college $48.8 \%$, Mid-Western regional hospital $18.9 \%$ and Okhaldhunga community hospital 9.5\%. This shows that CS rate was higher in urban part and in Medical College than in remote part and government hospital. In a retrospective study conducted in Gandaki medical college the CS rate was $41.26 \%$ and in a study done in Rajasthan CS rate was 31.1\%. ${ }^{11,12}$ The average global CS rate is $18.6 \% \%^{2}$

Out of total CS 248 patient underwent elective LSCS whereas 922 women underwent emergency LSCS. This disparity was due to high number of referral cases who had obstetrics or medical complication necessitating emergency cesarean section. Similar finding was noted in several studies. ${ }^{11,12,15,20 .}$

Majority of women were in the age group of 21-30 years. In developing country like ours the average age of marriage is late teen or early 20s. Most fertile period in women's life is also in their 20s. Because of this factors women undergoing cesarean section is also high in between 21-30 years.

The most common indication of caesarean section in our study was fetal distress $23.7 \%$ followed by previous cesarean section $23 \%$. Increase use of cardiotocographic monitoring of fetal heart rate leading to early detection of abnormal pattern, increase cases of postdated pregnancy and high risk cases with meconium stained liquor as a complication, unavailability of fetal scalp blood sampling to identify true distress are some of the reasons for increased incidence of caesarean done for fetal distress. Similar finding was seen in a study done in GMC where 25\% of cases underwent CS for fetal distress. ${ }^{11}$ In studies conducted in different institutes, though the main indication of performing caesarean was fetal distress the incidence varied like $40.2 \%$ in Kirtipur hospital, ${ }^{13} 12.5 \%$ in Ethopia, ${ }^{14} 15.44 \%$ in Sharif medical college $\mathrm{e}^{15}$ and $13 \%$ in Jhalawar medical college $\mathrm{e}^{12}$ respectively. Birla $S$ in her comparative study of indications of primary caesarean section between primigravida and multi gravida found that fetal distress was the most common indication in both group $(32.21 \%$ vs $17.41 \%) .{ }^{16}$ 
Most common maternal indication was previous cesarean section which constitutes $23 \%$ of cases. Similar finding was noted in a study done by Maimoona et $\mathrm{al}^{15}$ and Poudel et $\mathrm{al}^{8}$ where the incidence was $22.76 \%$ and $25.3 \%$ respectively. It is higher than in a study done in Ethiopia where the incidence was $18.9 \%{ }^{14}$ and in GMC $14 \%{ }^{11}$ and lower than in study done by Sharma et al $35 \% .{ }^{12}$

This increase in previous caesarean section cases can be due to a tendency to do elective or emergency repeat caesarean rather than going for trial of labour for vaginal birth after caesarean. This low threshold for performing VBAC is probably due to fear of uterine rupture in labour which is 5.2/1000 compared with (1.6/1000) in elective repeat caesarean delivery and this can be catastrophic leading to perinatal death $(1 / 1000)$ and very rarely maternal death. ${ }^{16-19}$ Cephalopelvic disproportion as indication was present in $15.6 \%$ which is lower than in a study done by Gurung et al $28 \% .^{11}$

The alarmingly increasing rate of caesarean needs to be addressed urgently from all the health sectors. Scientifically tested Strategies to promote natural birth and reducing CS should be incorporated in health policy. From government level introduction of strong mechanism to monitor caesarean section rate in both government and private sector need to be done. Expansion of midwifery profession to ensure 24 hour coverage in rural part of Nepal and more training on skilled birth attendance to promote natural birth needs to be focused. Institutions should form strong protocols for management of both complicated and uncomplicated deliveries and regular meeting needs to be held to ensure its implementation. Evidence based counseling to expecting couple for the better health outcome while planning the mode of delivery from obstetrician part should be done.

\section{CONCLUSION}

The study depicts that the rate of caesarean section rate was quite high in the institute and majority of the patients underwent an emergency cesarean section. The commonest indications of cesarean section were fetal distress and previous LSCS. As most cesarean section are currently based on physician's judgment, the study results depict need to develop and implement guidelines for performing cesarean section so that unjustified surgical delivery can be minimized. As the study is based on a single hospital, the results may not be generalizable. Hence, there is need for standardized collection of information on incidence and indications of cesarean section nationally so that comparison and improvements can take place. Additionally, further studies are recommended for understanding of causes and consequences of prevalence of high caesarean section rates.

\section{ACKNOWLEDGEMENT}

We would like to thank the hospital administration for allowing us to conduct the study in the hospital. We also like to acknowledge the help received from our students.

\section{CONFLICT OF INTEREST}

We declare no conflict of interest

\section{REFERENCES}

1. World Health Organization. Appropriate Technology For Birth. Lancet. 1985: WHO Statement on Caesarean Section Rates.2014; 2:436-437. https://doi.org/10.1016/S0140-6736(85)92750-3

2. Betran AP, Jian Feng $Y$, Moller $A B$, Jun Z, Gulmezoglu AM and Torloni MR. The Increasing Trend In Caeserean Section Rate: Global, Regional And National Estimates; 1990-2014.PLoS ONE.2016;11(2);e0148343.

https://doi.org/10.1371/journal.pone.0148343

3. Lumbiganon P, Laopaiboon M, Gulmezoglu AM, Souza JP, Taneepanichskul S, Ruyan P, et al. Method Of Delivery And Pregnancy Outcomes In Asia: The WHO Global Survey On Maternal And Perinatal Health 2007-2008. Lancet. 2010; 375(9713):490-499.

https://doi.org/10.1016/S0140-6736(09)61870-5

4. Souza JP, Gulmezoglu A, Lumbiganon P, Laopaiboon M, Carroli G, Fawole B, et al. Caesarean Section Without Medical Indications Is Associated With An Increased Risk Of Adverse Short-Term Maternal Outcomes: The 2004-2008 WHO Global Survey on Maternal and Perinatal Health. BMC Medicine. 2010; $8: 71$.

https://doi.org/10.1186/1741-7015-8-71

5. Villar J, Valladares E, Wojdyla D, Zavaleta N, Carroli G, Velazco A, et al. Caesarean Delivery Rates And Pregnancy Outcomes: The 2005 WHO Global Survey On M aternal And Perinatal Health. 2005 WHO Global Survey Maternal Perinatal Health. 2006;1819-1829. https://doi.org/10.1016/S0140-6736(06)68704-7

6. Mi J and Liu F. Rate of Cesarean Section Is Alarming In China. Lancet. 2014; 383(9927): 1463-1464.

https://doi.org/10.1016/S0140-6736(14)60716-9

7. Cavallaro FL, Cresswell JA, França GV, Victora CG, Barros AJ, et al. Trends In Cesarean Delivery By Country And Wealth Quintile: Cross-Sectional Surveys In Southern Asia And SubSaharan Africa. Bull World Health Organ. 2013; 91(12): 914-922. https://doi.org/10.2471/BLT.13.117598

8. Poudel R, Dangal G, Karki A, Pradhan H, Shrestha R, Bhattachan K, et al. Assessment Of Caesarean Section Rate At Kathmandu Model Hospital Using The Robson's Ten Group Classification System. Journal of Nepal Health Research Council. 2019; 17(45):491-494. https://doi.org/10.33314/jnhrc.v17i4.2117

9. Pradhan B, Shrestha SD, Sharma P and Bhandari S. Increasing Trend Of Caesarean Section In Patan Hospital. Journal Of 
General Practice And Emergency Medicine Of Nepal.2015; 6

10. Dhakal S, Regmi P, Teijlingen EV, Wood J, Dangal G, et al. Rising Rate of Caesarean Section in Urban Nepal. Journal of Nepal Health Research Council. 2018; 16(41): 479-480. https://doi.org/10.33314/jnhrc.v16i41.1750

11. Gurung RT, Gurung G, Shrestha R, Gurung $T$ and Sharma $P$. Prevalence And Outcome Of Cesarean Section At Gandaki Medical College Teaching Hospital And Research Centre. Journal of Gandaki Medical College.2016; 9(2). https://doi.org/10.3126/jgmcn.v9i2.17858

12. Sharma $\mathbf{N}$ and Jhanwar A. Study Of Incidence, Trends And Determinants Of Caesarean Section In Tertiary Care Hospital Of Rajasthan, India. Int J Reprod Contracept Obstet Gynecol. 2018; 7(7):2672-2676.

https://doi.org/10.18203/2320-1770.ijrcog20182504

13. Pradhan P, Shrestha S, Rajbhandari PK and Dangal G. Profile Of Caesarean Section In Kirtipur Hospital. Nepal Journal Of Obstetrics And Gynaecology.2014; 18(2):51-54. https://doi.org/10.3126/njog.v9i2.11763

14. Moges A, Beyene, Ademe BW and Akessa GM. Prevalence and Outcome of Caesarean Section in Attat Hospital Gurage Zone, SNNPR, Ethiopia. Archives of Medicine. 2015; 7(4):8.
15. Hafeez M, Yasin A, Badar N, Pasha Ml, Akram N and Gulzar B. Prevalence and Indication Of Caesarean Section In A Teaching Hospital. JIMSA. 2014; 27(1):15-16.

16. Birla S, Gupta M, Birla $P$ and Sharma J. Comparison Of Incidence, Indication And Complication Of Primary Cesarean Section In Primigravida And Multigravida. International Journal of Medical Science and Education.2016; 3(3):311-317.

17. Lydon-Rochelle M, Holt VL, Easterling TR and Martin DP. Risk of uterine Rupture during labour among women with a prior caesarean delivery. N Engl J Med. 2001; 345(1):3-8. https://doi.org/10.1056/NEJM200107053450101

18. Mozurkewick EL and Hutton EK. Elective repeat caesarean delivery versus trial of labour: A metaanalysis of literature from 1989 to 1999. Am J Obstet Gynecol. 2000; 183(5):1187-1197. https://doi.org/10.1067/mob.2000.108890

19. Rageth JC and Grossenbecher H. Delivery after previous caesarean: A risk evaluation. Swiss working group of Obstetrics and gynaecologic Institutions. Obstet Gynecol. 1999; 93(3):332-337. https://doi.org/10.1016/S0029-7844(98)00446-3

20. Latif R, Rafique $S$, Ashfaq M, Yasmeen T, Javaid S, et al. An Analysis Of Prevalence And Indications Of Caesarean Section In Primigravida. PJMH.2017; 11(1):9-11.

\section{Author's Contribution:}

AS - Concept and Design of the study, Interpretation of results, Preparation of manuscript and Revision of manuscript; JS - Concept and coordination of study, revision of manuscript; SDG - Review of literature, Revision of manuscript.

\section{Work attributed to:}

Department of Obstetrics and Gynecology, Manipal teaching hospital, Pokhara, Nepal.

Orcid ID:

Dr. Aashika Shrestha - io https://orcid.org/0000-0002-2738-6172

Dr. Junu Shrestha - (D) https://orcid.org/0000-0002-8683-2689

Dr. Sangeeta Devi Gurung - (1) https://orcid.org/0000-0002-3288-1856

Source of funding: None, Conflict of Interest: None. 